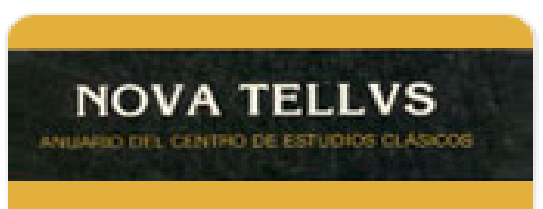

Nova Tellus

ISSN: 0185-3058

novatelu@servidor.unam.mx

Centro de Estudios Clásicos

México

González Gallardo, María Fernanda

Una tesis novohispana de 1668 sobre decuriones y regidores: texto, traducción y comentarios

Nova Tellus, vol. 32, núm. 2, 2015, pp. 289-305

Centro de Estudios Clásicos

Distrito Federal, México

Disponible en: http://www.redalyc.org/articulo.oa?id=59139884009

- Cómo citar el artículo

- Número completo

- Más información del artículo

- Página de la revista en redalyc.org

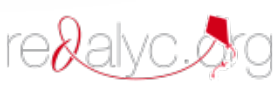

Sistema de Información Científica

Red de Revistas Científicas de América Latina, el Caribe, España y Portugal Proyecto académico sin fines de lucro, desarrollado bajo la iniciativa de acceso abierto 


\title{
Una tesis novohispana de 1668 sobre decuriones y regidores: texto, traducción y comentarios ${ }^{1}$
}

A 1668 Thesis on Decurions and Alderman of New Spain:

Text, Translation and Commentary

\author{
María Fernanda GonZÁlez GALlARDO \\ Universidad Nacional Autónoma de México \\ gonllardo@gmail.com
}

\begin{abstract}
Resumen: José Osorio Espinosa de los Monteros, quien llegó a ser Oidor de la Real Audiencia de México, obtuvo el grado de licenciado en Derecho civil en la Real Universidad de México con una tesis publicada en 1668 que se conserva en el Archivo General de la Nación (AGN). Se presenta en este trabajo el folio de la tesis, así como su transcripción paleográfica y traducción, antecedidos de una presentación y seguidos por un análisis filológico e histórico.
\end{abstract}

Abstract: Jose Osorio Espinosa de los Monteros, who became Judge of the Royal Court of Mexico, attained a degree in Civil Law from the Royal University of Mexico with a thesis published in 1668, and now preserved at the Nation's General Archive (AGN). The purpose of this paper is to present said thesis along with its paleographic transcription and his translation, as well as a philological and historical analysis.

Palabras clave: Tesis novohispanas, Real Universidad de México, Derecho novohispano, tradición clásica.

KEYwORDs: Novohispanic Thesis, Royal University of Mexico, Novohispanic Right, Classical Tradition.

RECIBIDO: 1 de octubre de 2014 - ACEPTADO: 18 de febrero de 2015.

\section{Presentación}

El Archivo General de la Nación resguarda las tesis de licenciados y doctores en Leyes de la Real Universidad de México, específicamente en la serie Universidad, volúmenes 277 y 278 . El estudio de estos documentos arroja datos importantes sobre la presencia del mundo clásico en

${ }^{1}$ El presente trabajo es parte de la investigación que realicé en la Maestría en Letras de la UNAM: "Las tesis de licenciados y doctores en Leyes de la Real Universidad de México en el siglo XVII: Código", mayo de 2014. Asimismo, una versión resumida de este texto se presentó en el XXVI Encuentro de Investigadores el Pensamiento Novohispano, el 6 de noviembre de 2013, en el Aula Magna del Instituto de Investigaciones Filológicas de la UNAM. 
la Nueva España, la historia de la Universidad, la historia del Derecho novohispano y la historia de México, en general.

En la Facultad de Leyes de la Real Universidad de México, inaugurada en 1553, se estudiaba Derecho civil a partir del Corpus Iuris Civilis, compilación de jurisprudencia clásica y material legislativo que Justiniano, emperador de Oriente, mandara redactar entre los años 528 y 533, compuesto de Institutiones o Instituta, Digesta o Pandectae, Codex, y Novellae.

El método que se aplicaba para el estudio del Derecho romano era el mos italicus o bartolismo jurídico, ${ }^{2}$ en el que el Derecho romano era considerado como ratio scripta incuestionable, y vera philosophia, por lo que no necesitaba del auxilio de otras disciplinas.

Para obtener los grados de licenciado o doctor, los alumnos debían presentar en lengua latina dos exámenes o actos de repetición, uno privado, en el que disertaban acerca del Digesto (Digesta), y otro público, en el que trataban y hacían tesis — también en latín- sobre el Código (Codex), libro integrado por constituciones imperiales que van desde el emperador Adriano (76-138) hasta Justiniano.

En la asignación de los pasajes o "puntos" de los exámenes se mezclaban azar y elección: ${ }^{3}$ se elegía a alguien "no letrado" para que abriera al azar el libro del Digesto o del Código en tres partes y efectuara tres "piques" consecutivos con un cuchillo; el primero de estos cortes lo daba en medio, y los otros dos a los lados. De estas tres partes, el aspirante al grado escogía un "punto general" acerca del cual se le señalaba una cuestión concreta o "punto más especial", repitiendo la operación para cada uno de los tres casos. ${ }^{4}$

Para el examen privado ${ }^{5}$ el sustentante debía preparar los puntos asignados y decirlos de memoria ante los sinodales, posteriormente debía contestar a tres réplicas en forma oral. El jurado, presidido por el doctor decano de la facultad y tres sinodales, determinaba la posibilidad de que se presentara o no el examen público, para el cual era necesario imprimir las tesis también llamadas "conclusiones" o "teoremas". Antes de imprimirlas el rector debía dar su visto bueno.

${ }^{2}$ Llamado así por el jurista Bártolo de Sassoferrato,

${ }^{3}$ Debido a estas variables, es posible intentar identificar las causas de la elección en el entorno sociopolítico.

${ }^{4}$ Cerralvo, 23, 6; Palafox 1775, p. 278.

${ }^{5}$ Cf. Carreño 2004. 
A diferencia de las tesis que hoy elaboramos para obtener grados universitarios, ${ }^{6}$ las tesis novohispanas consistían en una hoja de 25 a 45 centímetros de largo por 15 a 30 centímetros de ancho, estaban impresas por un solo lado, tenían frecuentes alusiones a personajes y conceptos de la cultura clásica y hacían referencia a personajes o conceptos de la cultura cristiana. En cuanto a su ornamentación, conforme transcurrieron los siglos del XVI al XVIII, fueron cada vez más decoradas, no sólo en los grabados, sino también en la tipografía. Su estructura puede dividirse en cinco partes:

1. En la parte superior se incluye el escudo del padrino (el virrey, un arzobispo o un personaje poderoso) y una dedicatoria, o la advocación a un santo o el escudo de una orden religiosa o un escudo de armas.

2. En seguida, el nombre del graduado y su ocupación, una especie de curriculum del aspirante.

3. Casi a la mitad de la hoja, la mención del pasaje del Corpus sobre el que el aspirante discurre.

4. Después, hasta cinco conclusiones, tesis o teoremas, deducidos del pasaje.

5. Por último, una doxología o fórmula de alabanza a la divinidad, el nombre del rector, el pie de imprenta (ciudad, impresor y año), y la firma del presidente del jurado.

En este trabajo se presentan la transcripción paleográfica, ${ }^{7}$ la traducción y un estudio filológico e histórico de la tesis de José Osorio Espinosa de los Monteros. En la transcripción paleográfica se desataron las abreviaturas æ, œ, el signo \&, las nasales finales $(n, m)$ y las partículas enclíticas (-que). Los corchetes [ ] se usaron para marcar las integraciones, mientras que las antilambdas $<>$ para conjeturas o reconstrucción

${ }^{6}$ El cambio en la forma de obtención de grados debió suceder en el paso de la Real Universidad de México a la Universidad Nacional Autónoma de México, inaugurada en 1910 por el presidente Porfirio Díaz, siendo Justo Sierra el profesor que fijara los ejes rectores.

${ }^{7}$ No se habla sólo de "paleografía" ya que ésta se aplica, en sentido estricto, a la transcripción de manuscritos. Los textos que se analizaron fueron impresos hace cuatro siglos. Lo que se hizo, surcando las dificultades, fue una "transcripción paleográfica", necesaria por el estado en el que se encuentran los documentos, el uso de abreviaturas, así como tipos que no corresponden al alfabeto latino. 
del texto. En caso de "errores" se consignó a pie de página la variante, como cuando en lugar de $V$ mayúscula se encuentra la letra $U$, en cuyo caso se mantuvo la segunda. En lo demás se respetó la ortografía del original y se conservaron las cursivas y las mayúsculas del texto latino, incluso en la traducción, con la finalidad de constatar su esencia ornamental y buscar, en la medida de lo posible, un impacto como el que pudo haber tenido en el siglo XVII. 


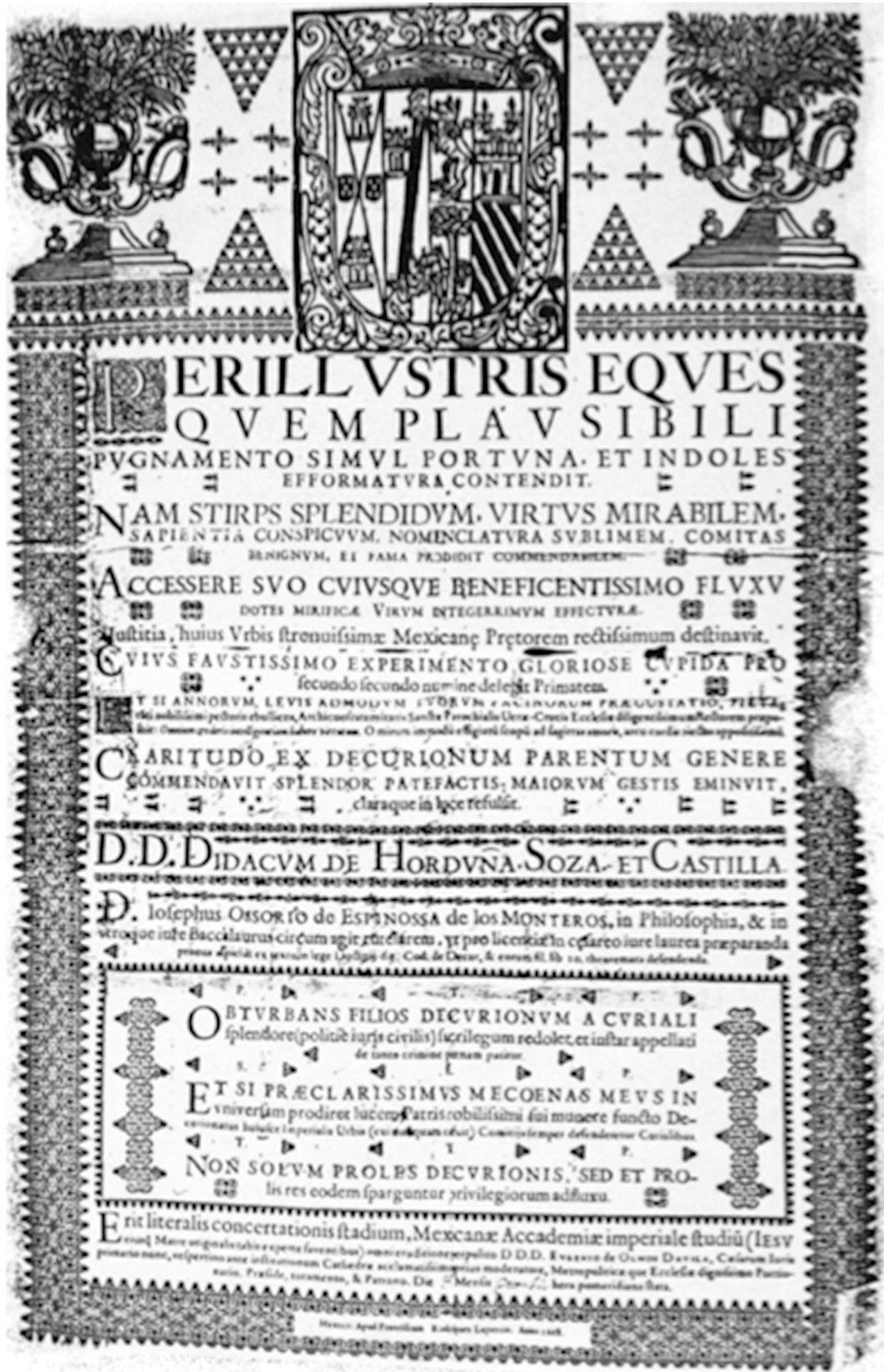


b) Transcripción paleográfica

\author{
PERILLVSTRIS EQVES \\ QVEM PLAVSIBILI \\ PVGNAMENTO SIMVL FORTVNA ET INDOLES \\ EFFORMATVRA CONTENDIT. \\ NAM STIRPS SPLENDIDVM, VIRTVS MIRABILEM, \\ SAPIENTIA CONSPICVVM, NOMENCLATVRA SVBLIMEM, COMITAS \\ BENIGNVM, ET FAMA PRODIDIT COMMENDABILEM. \\ ACCESSERE SVO CVIVSQVE BENEFICENTISSIMO FLVXV \\ DOTES MIRIFICAE VIRVM INTEGERRIMVM EFFECTVRAE. \\ Iustitia, huius Vrbis strenuissimae Mexican[ae] \\ $\operatorname{Pr}$ [ae]torem rectissimum destinavit.
}

CVIVS FAVSTISSIMO EXPERIMENTO GLORIOSE CVPIDA PRO

secundo secundo numine delegit Primatem.

ET SI ${ }^{8}$ ANNORVM, LEVIS ADMODVM SVORUM

FACINORVM PRAEGVSTATIO, PIETAS

ta[n]ti nobilissimi pectoris ebulliens, Archiconfraternitatis Sanctae Parochialis Verae-Crucis Ecclesiae diligentissimum Rectorem praeposuit:

Omnium crederis intelligentiam habere virtutum..$^{9} \mathrm{O}$ mirum immodu[m] effigiatu[m] scopu[m] ad sagittas amoris, arcu cordis

piectas $^{10}$ appositissimu[m].

CLARITUDO EX DECVRIONUM PARENTUM ${ }^{11}$ GENERE

COMMENDAVIT SPLENDOR PATEFACTIS MAIORVM GESTIS

EMINVIT,

claraque in luce refulsit.

D[IGNISSIMVM] D[OMINVM] DIDACVM DE HORDVÑA, SOZA ET CASTILLA.

D[ominus] Iosephus OSSORIO de ESPINOSSA de los Monteros, in Philosophia,

et in utroque iure Baccalaurus circumagit tutelarem, ut pro licentiae in c[ae]sareo iure laurea praeparanda

\footnotetext{
${ }^{8}$ ETSI

${ }^{9}$ Cassiod. Var. 5, 21.

${ }^{10}$ pietas

${ }^{11}$ CLARITVDO EX DECVRIONVM PARENTVM
} 
pronus aspiciat ex textu in lege Doctitii $63 .{ }^{12} \mathrm{Cod}[\mathrm{icis}] \mathrm{de}$ Decur[ionibus], et eorum fil[iis] lib[ro] $10 .{ }^{13}$ theoremata defendenda.

P[RIMVM] T[HEOREMA] P[OSSITVM] OBTVRBANS FILIOS DECVRIONVM A CVRIALI splendore (politiè iuris civilis) sacrilegum redolet et instar appellati de tanto crimine poenam patitur.

S[ECVNDVM] T[HEOREMA] P[OSSITVM] ET SI PRAECLARISSIMVS MECOENAS MEVS IN universam prodiret lucem Patris nobilissimi sui munere functo Decurionatus huiusce Imperialis Urbis (cui nu $<$ m $>$ quam cesuit) Comitiis semper defenderetur Curialibus.

\author{
T[ERTIVM] T[HEOREMA] P[OSSITVM] \\ NON SOLVM PROLES DECVRIONIS, SED ET PROLIS \\ res eodem sparguntur privilegiorum adfluxu.
}

Erit literalis concertationis stadium, Mexicanae Accademiae imperiale studiu[m] (IESV eiusq[ue] Matre originalis tabis experte faventibus) omni eruditione per polito D[IGNISSIMO] D[OMINO] D[ON] EVGENIO DE OLMOS DAVILA, Caesarum Iuris primario nunc, vespertino ante institutionum Cathedrae acclamatissimo prius moderatore, Metropoliticae que Ecclesiae dignissimo Portionario. Praeside, tutamento, et Patrono. Die 8 Mensis Decembris hora pomeridiana stata.

MEXICI: Apud Franciscum Rodirguez Lupercio. Anno 1668.

12 C. 10, 32, 63. Imperator Leo. Docticii viri clarissimi iuvenis fortuna super curiali condicione nullo modo inquietanda nec facultatibus eius, sed eo, qui huiusmodi conamen inierit, sacrilegii poenam luituro omnes, qui post peractam illustrem administrationem vel adhuc in ea posito patri suo giguntur, a curialium intentione defendantur una cum paternis sine dubio substantiis, quas cum persona patris a curiali nexu liberas esse nemo dissimulet. * LEO A. AD SENATVM. * $<$ A XXX $>$.

${ }^{13}$ C. 10, 32. De decurionibus et filiis eorum et qui decuriones habentur et quibus modis a fortuna curiae liberentur. 
c) Traducción

[Dedicatoria]

Ilustrísimo caballero para quien la fortuna y la naturaleza que ha de formarse se esfuerza con plausible pugna ya que su estirpe revela al espléndido, su virtud al admirable, su sapiencia al conspicuo, su nomenclatura al sublime, su cortesía al benigno y su fama al digno de recomendación. Las dotes maravillosas que deben completarse se acercaron al varón integérrimo por su beneficentísimo flujo.

La justicia deseosa (lo) destinó Pretor rectísimo de su Ciudad Mexicana diligentísima, de la que por favorabilísimo experimento gloriosamente delegó como el primer numen en lugar del segundo.

Por otra parte, la piedad de los años, prueba perfectamente leve de sus acciones, que brota de nobilísimo pecho, (lo) puso como Rector diligentísimo de la Confradía de obispos de la Santa Iglesia Parroquial de Veracruz: Creerás tener conocimiento de todas las virtudes. ¡Oh! ¡Increíblemente, el blanco de las flechas del amor es representado como la piedad, dispuestísimo con el arco del corazón!

La claridad recomendó al Dignísimo Señor Diego de Orduña, Sosa y Castilla a partir del origen de los padres decuriones, el esplendor se elevó con las gestas evidentes de los antepasados y refulgió en la clara luz.

[Datos del graduado]

El señor José Osorio Espinosa de los Monteros, Bachiller tutelar que se desarrolla en Filosofía y en ambos derechos, a favor de la licencia áurea en Derecho cesáreo que ha de ser preparada, observe inclinado estos teoremas que han de ser defendidos

[Pasaje]

a partir del texto en la ley 63 de Docticio del Código sobre los Decuriones y sus hijos <y quienes son tenidos por decuriones y por qué medios son liberados de la condición de la curia> en el libro 10. 
[Teoremas o conclusiones]

Primer teorema propuesto

El que molesta a los hijos de los decuriones en cuanto al esplendor curial (por gobierno de derecho civil) huele el sacrilegio y sufre la pena por tan gran crimen llamado.

Segundo teorema propuesto

SI mi preclarísimo mecenas naciera una vez realizado el trabajo de su nobilísimo Padre, el Decurión de esta Urbe Imperial (para la cual nunca cesó), siempre será defendido por los Comicios Curiales.

Tercer teorema propuesto

No sólo los hijos del decurión sino también las cosas del hijo son esparcidas por la misma afluencia de los privilegios.

[Datos finales]

Será un concurso de batalla literal, un estudio imperial de la Academia Mexicana (favoreciéndolo Jesús y su madre libre de la corrupción original) con toda la erudición a causa del refinado Dignísimo Señor Don Eugenio de Olmos Dávila, ahora el principal del Derecho Cesáreo, antes [maestro] vespertino de la cátedra de instituciones, primeramente moderador aclamadísimo y dignísimo Porcionero de la Iglesia Metropolitana. Presidente, defensa y patrono. En el día 8 del mes diciembre en la hora establecida después del meridiano.

México, en la casa de Francisco Rodríguez Lupercio. Año 1668.

\section{COMENTARIOS}

a) Los personajes

Marcelo da Rocha Wanderley afirma que "la conformación de una carrera tiene como fundamento la capacidad de convertir los contactos sociales en formas específicas de beneficio y reputación". ${ }^{14}$ Esta idea se ve confirmada por José Osorio Espinosa de los Monteros, quien supo construir una red social teniendo como aliado principal y mecenas a Diego de Orduña Sosa y Castilla, aristócrata hacendado y pío, que le facilitó

\footnotetext{
${ }^{14}$ Da Rocha 2004, p. 230.
} 
la obtención del grado de licenciado para posteriormente hacer carrera como abogado y llegar a ser Oidor de la Real Audiencia.

Diego de Orduña fue hijo de Juan de Orduña, capitán y sargento mayor "regidor" en México, y familiar del Santo Oficio. Su familia era de abolengo en México, instituyó mayorazgos y llegó a tener haciendas ganaderas; fue considerado vecino de la Ciudad de México. Entre los años 1671 y 1672 mandó edificar la capilla de la hacienda de San José Buenavista en Querétaro. Contrajo matrimonio con Teresa Cantabrana, hija de Domingo de Cantabrana, la cual, ya viuda, estuvo inmersa en un pleito por la posesión de una hacienda. ${ }^{15}$

Eugenio de Olmos Dávila, presidente del examen, fue nombrado abogado en 1644. Prestó servicios a la religión y fue catedrático y decano de la Real Universidad de México. Obtuvo la cátedra Prima de filosofía, y la cátedra de Vísperas de cánones. A partir de 1653 fue nombrado catedrático de Instituta. En 1655, por decreto del duque de Albuquerque, fue catedrático de Código (cátedra Vísperas de leyes) y en 1657 de la cátedra Prima de Leyes con el referendo del secretario de gobierno Pedro Velásquez de la Cadena. ${ }^{16}$

Francisco Rodríguez Lupercio fue impresor librero desde 1658. A su muerte, en 1683, continuó con la labor su viuda, Jerónima Delgado, quien imprimió esta tesis. De 1693 a 1736, el pie de imprenta de los textos era "herederos de la viuda de Francisco Rodríguez Lupercio". Toda la familia produjo aproximadamente 444 impresos. ${ }^{17}$

José Osorio, quien se licenció con esta tesis, era bachiller en Filosofía y Derecho. Hacia 1692 ya fungía como Oidor de la Real Audiencia, año en el que resultó herido junto con el fiscal de la Real Audiencia, don Luis Martínez Hidalgo Montemayor, y el capitán don Alonso Ramos de Herrera y Salcedo, a causa del enfrentamiento entre "gobernistas" (partidarios de don Gerónimo de Soria y don Francisco Sarmiento) y "clericales" (pertenecientes a la canonjía doctoral de la catedral de Guadalajara, en su mayoría estudiantes de la Compañía de Jesús). ${ }^{18}$ La relación entre José y su mecenas era bastante fuerte, pues escribió la que pareciera ser la defensa de su hermana, María Orduña: la Alegación por la justicia que asiste a Doña María Ordu-

\footnotetext{
${ }^{15}$ Fernández 1965, pp. 319-326.

${ }^{16}$ Da Rocha 2004, pp. 211-216.

${ }^{17}$ Martínez 2002, p. 13.

18 Villaseñor 1954, pp. 103-104.
} 
ña, Sosa y Castilla, muger del Maestre de Campo, D. Francisco Guerrero Ardila sobre un Mayorazgo, Imp. en Megico en fol. sin año. ${ }^{19}$

\section{b) Decuriones y regidores}

Si bien las tesis novohispanas no incorporaron elementos novedosos a la ciencia jurídica novohispana, porque el método de estudio del Derecho romano no permitía debatir las fuentes, sí actualizaron algunos términos del latín clásico. Tal es el caso de la palabra decurio.

$\mathrm{Al}$ referirse al padre del mecenas como Decurionatus huiusce Imperialis Urbis, José Osorio Espinosa de los Monteros traza en su tesis una línea entre Roma y Nueva España. Lo hace en las conclusiones, ${ }^{20}$ donde no es común encontrar alusiones a los involucrados en el examen, consiguiendo así darle mayor relevancia.

Para intentar comprender por qué se refiere así a él es pertinente hacer una revisión del término.

Históricamente, la palabra "decurión" tuvo varios significados que van desde el jefe de una curia como unidad táctica militar, el militar menor encargado de la caballería, o el consejero municipal. ${ }^{21}$ En la República, los decuriones eran elegidos entre las personas más ricas e ilustres del municipio por los duoviri iure dicundo. En el Imperio, se denominaron decuriones curiales, porque el senado municipal que constituían se llamaba también curia; sólo existían en las ciudades que tenían el carácter de municipios. Quienes eran o habían sido decuriones formaban una orden social especial. Posteriormente, el cargo se hizo muy costoso y la condición de los decuriones se volvió deplorable.

Si se compara la situación social "especial" de la que gozaban los decuriones con las condiciones de vida de los regidores en la Nueva España, se pueden encontrar similitudes.

Durante el siglo XVII, los decuriones o regidores debían ser vecinos de la capital y generalmente eran criollos, porque los peninsulares que

${ }^{19}$ Beristáin 1981, tomo II.

${ }^{20}$ S[ECVNDVM] T[HEOREMA] P[OSSITVM] ET SI PRAECLARISSIMVS MECOENAS MEVS IN universam prodiret lucem Patris nobilissimi sui munere functo Decurionatus huiusce Imperialis Urbis (cui $\mathrm{nu}<\mathrm{m}>$ quam cesuit) Comitiis semper defenderetur $\mathrm{Cu}$ rialibus.

${ }^{21}$ Cf. Le Bohec 2007, pp. 58-62. 
vivían en la ciudad de México ocupaban los puestos más altos. Los regidores elegían a los alcaldes ordinarios, quienes eran los jueces de primera instancia, y administraban la ciudad; cuidaban de los bienes de la misma, de las obras públicas y de su abasto; otorgaban licencias de trabajo a los artesanos, y vigilaban y controlaban el comercio en general; se preocupaban por la salud pública y por la educación elemental. ${ }^{22}$ Entre estas funciones podría colocarse la de ser consejero municipal, como el decurio romano.

Cuando se incrementó el número de los doce regidores del cabildo establecidos por la ley, ya porque se nombrara a un contador, un veedor y un factor de la Real Hacienda con voz y voto en el cabildo, los criollos lucharon por evitar abusos del poder y alcanzar los privilegios que les eran negados a quienes no habían nacido en España; ${ }^{23}$ supieron aprovechar su calidad de "descendientes de conquistador", título honorífico, para recibir del rey de España mercedes de tierras, con lo que se volvieron una "aristocracia rural", como la familia de Juan de Orduña.

José Osorio dice en sus teoremas que tanto los decuriones como sus hijos y sus cosas están protegidos por causas de Derecho civil y defendidos por los comicios curiales. El pasaje de donde se deduce esto corresponde al párrafo (o ley) 63 del título 31 del libro 10 del Código, en donde se lee:

Que la fortuna del varón Docticio, joven ilustrísimo, de ningún modo se haya de molestar por la condición curial ni por sus facultades, pues a aquel que lo hubiera intentado se le castigará con la pena del sacrilegio. Todos los que nacen al padre, después de llevada su administración o aún en ella, sean defendidos de la demanda de los curiales, sin duda, junto con los bienes paternos, que con la persona del padre, nadie ignore que están libres del nexo curial. $^{24}$

${ }^{22}$ Flores 1970, p. 6.

${ }^{23}$ Idem, p. 24.

${ }^{24}$ C., 10. 32. 63. Imperator Leo. Docticii viri clarissimi iuvenis fortuna super curiali condicione nullo modo inquietanda nec facultatibus eius, sed eo, qui huiusmodi conamen inierit, sacrilegii poenam luituro omnes, qui post peractam illustrem administrationem vel adhuc in ea posito patri suo giguntur, a curialium intentione defendantur una cum paternis sine dubio substantiis, quas cum persona patris a curiali nexu liberas esse nemo dissimulet. * LEO A. AD SENATVM. *<A XXX>. 
Como puede verse son casi las mismas palabras que repite José Osorio, salvo que éste incluye a su mecenas como uno más de los decuriones.

\section{c) Citas literarias}

Las tesis de la Facultad de Leyes no estaban exentas de literatura, cuestión que no debe extrañar si se toma en cuenta que todos los alumnos de la Real Universidad de México cursaban de manera preparatoria la Facultad de Artes en donde se enseñaban, entre otras materias, lenguas clásicas y literatura.

En la dedicatoria al mecenas Diego de Orduña se cita en letras cursivas el verso 21 de la Epístola 5 de Casiodoro, escritor latino que vivió bajo el reinado de Teodorico, rey de los ostrogodos y contemporáneo de Boecio, considerado uno de los transmisores de la cultura latina clásica en el cristianismo: Omnium crederis intelligentiam habere virtutum (“creerás tener conocimiento de todas las virtudes"). En la impresión no se incluye la referencia a la obra, tal vez por cierto descuido o porque José Osorio citó de memoria y no supo el lugar exacto. De cualquier modo, la cita sí tiene relación con la persona del mecenas, pues Diego de Orduña fue familiar del Santo Oficio y estos personajes debían contar con numerosas virtudes.

\section{d) Religión}

La presencia de la religión cristiana en el documento es reflejo fiel de la época. En este caso presenta características literarias.

O mirum immodum effigiatum scopum ad sagittas amoris, arcu cordis pietas appositissimum (“¡OH! ¡Increíblemente, el blanco de las flechas del amor es representado como la piedad, dispuestísimo con el arco del corazón!"). Esta exclamación hace referencia a Diego de Orduña, Archiconfraternitatis Sanctae Parochialis Verae-Crucis Ecclesiae diligentissimus Rector ("rector diligentísimo de la Cofradía de obispos de la Santa Iglesia Parroquial de Veracruz"). Puede entenderse que el punto en donde se clavan las flechas del amor es imaginado como la piedad. Si se toma en cuenta que el señor Diego mandó edificar la capilla de la hacienda de San José Buenavista entre los años 1671 y 1672, se tiene 
entonces que además de un personaje de la alcurnia mexicana, como oficial del Santo Oficio, él mismo es considerado la piedad.

A semejanza de otras tesis novohispanas, en la última parte se incluye la advocación a Dios Iesu eiusque Matre originalis tabis experte faventibus ("favoreciéndolo Jesús y su madre libre de la corrupción original"). Se trata de una doxología común o fórmula de alabanza a la divinidad, ${ }^{25}$ pues los estatutos universitarios establecían que tanto los que se graduaban de bachilleres, licenciados, doctores y maestros, como los que se incorporaban a la Universidad y llevaban cátedras debían hacer el juramento por la Inmaculada Concepción de la Virgen María. ${ }^{26}$

\section{e) Lengua}

Aunque la tesis no es muy compleja, el latín en que está escrita presenta elementos dignos de nota.

La pompa con la que se hace referencia a los personajes es característica de las tesis novohispanas y retrata el periodo barroco en que se vivía. Por razones obvias (el financiamiento de los estudios), se exalta más al padrino, Diego de Orduña, llamándolo perillustris eques, virum integerrimum, praetorem rectissimum, diligentissimum rectorem, dignissimum dominum, y praeclarissimus mecoenas, hijo de un patris nobilissimi. No se deja de lado a quien presidió el examen, Eugenio de Olmos Dávila, a quien se le llama dignissimo domino, aclamatissimo moderatore, dignissimo portionario. Este tono tiene gran actualidad; aún se nota en discursos de carácter político.

Puede apreciarse un matiz de guerra o competencia en todo el texto. La naturaleza plausibili pugnamento contendit ("se esfuerza con plausible pugna”) para el ilustrísimo caballero a quien va dedicada la tesis. Este personaje tiene un origen ex decurionum parentum ("a partir de los padres decuriones"), y se considera la defensa de las conclusiones como literalis concertationis stadium ("un concurso de batalla literal").

Se observa también el término theorema en vez de thesis o conclusio. Un "teorema" es una proposición no primitiva, pero derivada o

${ }^{25}$ Se trata de un punto importante, si se toma en cuenta que esta tradición se remonta a los salmos hebreos en los que se glorificaba y alababa a Jahvé. Cf. Brioso 1972, cap. 1 .

${ }^{26}$ Carreño 1961, p. 157. 
derivable de otras proposiciones. ${ }^{27} \mathrm{Al}$ no haber deducido sus "teoremas" de los pasajes del Código, sino haberlos copiado, se puede decir que los términos "teorema", "tesis" y "conclusión" eran usados indistintamente como sinónimos.

La frase caesareum ius se refiere al Derecho civil, y cuando se dice que alguien es bachiller o licenciado in utroque iure ("en ambos derechos") quiere decir que es bachiller o licenciado tanto en Derecho civil como en Derecho canónico.

La tesis presenta una errata, que podría fácilmente adjudicársele al impresor. Dice piectas en vez de pietas, "piedad".

\section{Reflexiones finales}

Los elementos analizados de la tesis de José Osorio Espinosa de los Monteros muestran las relaciones existentes entre la Real Universidad de México, la Iglesia, el gobierno y las imprentas de la época, además de las prácticas educativas y los problemas que se trataban en la Facultad de Leyes de esta institución. Por último, constatan el legado invaluable que nos vincula directamente con la cultura latina.

Para el análisis de este tipo de documentos, innumerables, es fundamental la interdisciplina: el Derecho, la Filología, la Historia e incluso la Estética. Promover los distintos acercamientos asegurará una mayor comprensión y enriquecerá los estudios de historia de la Universidad, la historia del Derecho novohispano y la historia de México.

${ }^{27}$ Abbagnano 1980, s.v. teorema. 


\section{BIBLIOGRAFÍA}

\section{Fuentes}

GonzÁlez GonzÁlez, Enrique, Proyecto de estatutos ordenados por el virrey Cerralvo (1626), México, Centro de Estudios sobre la Universidad, Universidad Nacional Autónoma de México, 1991 (La Real Universidad de México. Estudios y textos, III).

Palafox y Mendoza, Juan de, Constituciones de la Real y Pontificia Universidad de México, $2^{\mathrm{a}}$ ed., México, Imprenta de D. Felipe de Zúñiga y Ontiveros, 1775.

Serie Universidad, Archivo General de la Nación, vol. 277, fol. 437.

KrüGer, P. et Th. Mommsen, Corpus iuris civilis, editio stereotypa, Berlín, 1963.

\section{Obras generales}

Brioso SÁnchez, Máximo, Aspectos y problemas del himno cristiano primitivo, Salamanca, Consejo Superior de Investigaciones Científicas, 1972.

CArreño, Alberto María, La Real y Pontificia Universidad en México 1536-1865, México, Universidad Nacional Autónoma de México, Coordinación de Humanidades e IIH, 1961.

CARREÑo, Elvia, La imprenta y la Universidad, ADABI, 2004, en http://www.adabi. org.mx/content/ Notas. jsfx?id=851> [fecha de consulta: 30 de septiembre de 2014].

Da Rocha Wanderley, Marcelo, "Si saben ustedes de los méritos”, en Carrera, linaje y patronazgo: clérigos y juristas en Nueva España, Chile y Perú (siglos XVI-XVII), Rodolfo Aguirre Salvador (coord.), México, Plaza y Valdés / Universidad Nacional Autónoma de México, Centro de Estudios Sobre la Universidad, 2004, pp. 177-237.

FERnÁNDEZ DE RECAS, Guillermo, Mayorazgos de la Nueva España, México, Instituto Bibliográfico Mexicano / Universidad Nacional Autónoma de México, 1965, pp. 319-326.

Flores OlEA, Aurora, "Los regidores de la ciudad de México en la primera mitad del siglo XVII", en Estudios de Historia Novohispana, 3, México, Universidad Nacional Autónoma de México, IIH, 1970, pp. 1-24.

Le BoHec, Yann, El ejército romano, Ignacio Hierro (trad.), Barcelona, Ariel, 2007.

Martínez Leal, Luisa, "Los impresores libreros en Nueva España del siglo XVII", Revista Casa del Tiempo, México, UAM, 2002, pp. 22-31.

Vargas Valencia, Aurelia, Las Instituciones de Justiniano en Nueva España, México, Universidad Nacional Autónoma de México, IIFL, 2001.

VILLASEÑOR Bordes, Rubén, "Un obispo y un presidente de la Audiencia", Historia mexicana, 4, 1 (13), 1954, pp. 99-106. 
Diccionarios y enciclopedias

Abbagnano, Nicola, Diccionario de Filosofía, México, FCE, 1980.

BERISTÁIn de SouZA, José Mariano, Biblioteca Hispanoamericana Septentrional, 3 vols., México, Claustro de Sor Juana, 1980-1981.

Diccionario de la Lengua Española, 22ª ed., Madrid, RAE, 2001.

Enciclopedia Universal Ilustrada, tomo XVII, Madrid, Espasa-Calpe, 1976. 
